\title{
Temporal Characteristics and Radiative Properties of Uniform Mo and Combined with Al Triple Planar Wire Arrays
}

\author{
M.E. Weller, A.S. Safronova, V.L. Kantsyrev, I. Shrestha, A. Stafford, \\ V.V. Shlyaptseva, E.E. Petkov \\ Physics Department, University of Nevada, Reno, NV 89557, USA
}

\begin{abstract}
Results of the research with triple planar wire arrays (TPWAs) made of uniform Mo or from combined Mo and Al planes obtained on the University of Nevada, Reno (UNR) Zebra generator are presented. The combined loads consisted of either two Mo planes on the outside with $\mathrm{Al}$ in the center or two Al planes on the outside with Mo in the center. Different wire diameters of Mo and Al were used to keep the planes approximately the same mass Also, the change of inter-planar gap was investigated: decreasing the interplanar gap from $3.0 \mathrm{~mm}$ to $1.5 \mathrm{~mm}$ led to a higher yield of $25 \mathrm{~kJ}$ (up from $16 \mathrm{~kJ}$ ), close to the highest yield from Mo double planar wire arrays. The question of how Mo and Al plasmas mix is raised and is examined and as a result it is demonstrated that L-shell Mo $(\sim 1000 \mathrm{eV})$ and $\mathrm{K}$-shell $\mathrm{Al}(\sim 400 \mathrm{eV})$ plasmas have very different electron temperatures. Additionally, time-gated $\mathrm{K}$-shell $\mathrm{Al}$ and Mg plasma parameters were modeled, giving important information on the time-evolution of such plasmas. The future work on radiation from mixed higher and lower atomic number wire arrays is discussed.
\end{abstract}

PACS: 52.58Lq; 52.59.Qy; 32.30.Rj; 52.70.La

\section{Introduction}

Planar wire arrays (single and multi-planar) have previously shown to be very efficient x-ray radiators $[1,2]$ on university scale z-pinch generators, such as the 1.7 MA Zebra generator at UNR. In 2007, a study [3] that involved mixing Mo and Al in single planar wire arrays (SPWAs) was accomplished and it was shown that L-shell Mo radiated mostly from random bright spots along the pinch, while K-shell Al radiated in a more column-like manner along the pinch. In continuation of this work on combined Mo and Al PWAs, single and double planar wire arrays of Mo were studied [4] and it was shown that Mo SPWAs produced very high $T_{e}$ at $>1300 \mathrm{eV}$ for heavier loads $(150 \mu \mathrm{g})$ and moderately high electron density $\left(n_{e}\right.$ at $\left.>10^{21} \mathrm{~cm}^{-3}\right)$ for lighter loads $(90 \mu \mathrm{g})$ with total radiated energies $>$ $18 \mathrm{~kJ}$. Mo double planar wire arrays (DPWAs), however, produced lower electron temperature $\left(T_{e}\right.$ at $>$ *Corresponding Author.

E-mail address: mweller@unr.edu (M.E. Weller) 
$1100 \mathrm{eV}$ ) but with higher total radiated energies $>21 \mathrm{~kJ}$. These studies motivated research into applying new methods into understanding radiation from different materials in wire array plasmas, and recently in [5] a new method was suggested that involved multi-planar wire arrays, in particular triple planar wire arrays (TPWAs), which provide the initial space separation of different plasmas and the possibility of their observation. In that work, $\mathrm{Cu}$ and $\mathrm{Al}$ wires were employed in which $\mathrm{K}$-shell $\mathrm{Al}$ and $\mathrm{L}$-shell $\mathrm{Cu}$ radiated at very similar electron temperatures and plasma conditions. The next step then, which this paper focuses on, is to study wires using materials that radiate at much different electron temperatures to show more how different plasmas mix, which L-shell Mo and K-shell Al accomplish. Studying combined plasmas with different initial mass concentrations can also be used to study opacity effects, which is important when trying to identify plasma parameters. Therefore, this paper will concentrate on the radiative characteristics of Mo and mixed Mo and Al triple planar wire arrays (TPWAs), in particular how geometry and inter-planar gaps play a role in efficiency of energy conversion to x-rays, and also how placement and mass concentration of different Mo and $\mathrm{Al}$ wires influence overall radiation of K-shell Al and L-shell Mo, and how this affects opacity of the plasmas. Section II describes experimental details and temporal characteristics of the TPWAs. Section III explores the radiative characteristics of the TPWAs. Section IV concludes the paper.

\section{Experimental Details and Temporal Characteristics of Mo and Combined with Al TPWAs}

The experimental details of the Mo and combined Mo and Al TPWAs are laid out in Table 1. All experiments contained six wires in each plane with an anode-cathode gap of $20 \mathrm{~mm}$, and were carried out on the Zebra generator at 1.0 MA configuration (100 ns current rise, $150 \mathrm{~kJ}$ stored energy). The first set of experiments, shot numbers 1261, 1262, and 1263, were carried out with an inter-planar gap of 3.0 $\mathrm{mm}$. The second set of experiments, shot numbers 1969, 1932, 1968, 1933, 1953, and 2166, were carried out with an inter-planar gap of $1.5 \mathrm{~mm}$. The TPWAs can be characterized into three categories: the first consisting of uniform $\mathrm{Mo}$ (Mo/Mo/Mo), the second consisting of $\sim 67 \% \mathrm{Mo}$ and $\sim 33 \% \mathrm{Al}(5056,95 \% \mathrm{Al}$, $5 \% \mathrm{Mg})(\mathrm{Mo} / \mathrm{Al} / \mathrm{Mo})$, with Mo planes on the outside, and the third consisting of $\sim 33 \% \mathrm{Mo}$ and $\sim 67 \% \mathrm{Al}$ (5056) (Al/Mo/Al), with Al planes on the outside. The wire thicknesses were adjusted to not only ensure that each plane had approximately the same mass, but that the load would implode at approximately 100 ns, or the maximum of the current, which allows maximum efficiency of x-ray radiation and total radiated energy output. Al 5056 alloy wires are utilized to help in countering opacity effects for K-shell Al as suggested in Ref. [6]. 
For the TPWAs with a $3.0 \mathrm{~mm}$ inter-planar gap, the total radiated energy for the $\mathrm{Mo} / \mathrm{Mo} / \mathrm{Mo}$ and $\mathrm{Mo} / \mathrm{Al} / \mathrm{Mo}$ loads were comparable at 16.2 and $16.9 \mathrm{~kJ}$, respectively. The total radiated energy for $\mathrm{Al} / \mathrm{Mo} / \mathrm{Al}$ load, however, was less at $13.1 \mathrm{~kJ}$. These energies are less than for the Mo DPWAs (> $21 \mathrm{~kJ})$ previously studied in Ref [4] and it is important to note here that because of the open magnetic configuration of the TPWA the placement of the outer wires can significantly change the dynamics and efficiency of the implosion. In order to attain higher efficiency in total radiation output, experiments were carried out by changing the inter-planar gap from $3.0 \mathrm{~mm}$ to $1.5 \mathrm{~mm}$. By doing so this makes the total outer-planar gap $3.0 \mathrm{~mm}$, which is the same as the outer-planar gap of the Mo DPWAs with the highest total radiated energy that were previously studied. Looking back to Table 1 , it can be seen that the TPWA loads with a $1.5 \mathrm{~mm}$ inter-planar gap have increased total radiated energy. The Mo/Mo/Mo loads radiated $25.5 \mathrm{~kJ}$ and $24.5 \mathrm{~kJ}$, the $\mathrm{Mo} / \mathrm{Al} / \mathrm{Mo}$ loads radiated $22.2 \mathrm{~kJ}$ and $22.5 \mathrm{~kJ}$, and the $\mathrm{Al} / \mathrm{Mo} / \mathrm{Al}$ loads radiated $23.1 \mathrm{~kJ}$ and $24.0 \mathrm{~kJ}$ of total energy, an increase of an average 1.6 times the previous values, and show good reproducibility to within 5\%. Similar results from DPWAs were attained in Ref [2] where it was shown that when comparing inter-planar gaps between $1.5 \mathrm{~mm}$ and $6.0 \mathrm{~mm}$, the maximum total radiated energy corresponded to the $1.5 \mathrm{~mm}$ inter-planar gap. A possible explanation is that when the initial plane separation is too far apart the load will be more difficult to implode. This will increase the implosion time and by doing so the implosion will not utilize the maximum of the current which may decrease the total radiated energy. Reducing the total mass can compensate for the larger gap; however this results in less mass that can radiate which may also decrease total radiated energy. Therefore there needs to be a balance between inter-planar gap and total mass. The results here indicate that for TPWAs the inter-planar gap of $1.5 \mathrm{~mm}$ provides optimal results, similar to Ref [2] with DPWAs. To compare radiated energy in the $\mathrm{x}$-ray regime, a calibrated photoconduction diode (PCD) was filtered ( $8 \mu \mathrm{m} \mathrm{Be},>$ $0.8 \mathrm{keV}$ ) to include both $\mathrm{K}$-shell $\mathrm{Al}$ and L-shell Mo radiation. The PCD energies for the $3.0 \mathrm{~mm}$ interplanar gap loads radiated between $102-179 \mathrm{~J}$, while the PCD energies for the $1.5 \mathrm{~mm}$ inter-planar gap loads radiated between $157-349 \mathrm{~J}$, depending on the Al concentration. Comparing loads with similar Al concentration and placement, there is an increase in PCD energy from roughly $1.5-2$ times when going from a $3.0 \mathrm{~mm}$ to $1.5 \mathrm{~mm}$ interplanar gap, which agree with the increase in total radiated energy. The fraction of x-ray radiation within the PCD cutoff energy range to the total radiated energy is between 0.6 $-1.5 \%$, indicating a vast majority of the radiation comes from softer radiation. Additionally, it can be noted that PCD energy appears to increase with increasing Al concentration, possibly because it's easier to radiate into K-shell Al than into L-shell Mo. The remainder of the paper will focus on these loads with a $1.5 \mathrm{~mm}$ inter-planar gap, in particular shots 1969, 1968, and 1953 (their respective identical loads 1932, 1933, and 2166 will not be shown because their time-resolved and radiative characteristics were similar).

The rest of this section goes over the experimental results of the temporal dynamics of the 
TPWAs with a $1.5 \mathrm{~mm}$ inter-planar gap in order to better understand how the different configurations implode and also how K-shell Al radiates in time. Figure 1 shows signals, current, and time-gated pinhole (TGPH) images from Mo/Mo/Mo (1969). The signals include a $5 \mu \mathrm{m}$ Kimfoil filtered x-ray diode $(\mathrm{XRD},>0.2 \mathrm{keV}$ ) and a calibrated PCD, as mentioned earlier. Briefly, the TGPH utilized a microchannel plate consisting of six gates with 6 ns spacing between gates and 3 ns frame duration, allowing two images of energies $>1.0 \mathrm{keV}$ and $>3.0 \mathrm{keV}$. The XRD signal indicates a sharp rise just before the implosion at $94 \mathrm{~ns}$, followed by a series of bursts after the main pinch lasting for approximately 40 ns. The PCD signals show interesting results where, similar to the XRD signals, there are a series of bursts and the start and the end is virtually identical to the timing of the XRD. The TGPH images indicate many random bright spot formations for both $>1.0$ and $>3.0 \mathrm{keV}$ images, which correlate well with the random burst nature of the XRD and PCD signals. The bright spot formations have been shown in other wire array configurations [7-10] to correlate with Rayleigh Taylor instabilities which are seeded by the development of quasi-periodic gaps in the wire cores at the start of the implosion phase, that may occur also in these experiments.

Next in Figure 2 are the signals and time-gated spectrometer (TGSP) images from Mo/Al/Mo (1968). The TGSP [11] was implemented to understand how $\mathrm{K}$-shell $\mathrm{Al}$ and $\mathrm{Mg}$ radiate in time and utilized a potassium hydrogen phthalate $(\mathrm{KAP})$ crystal $(2 \mathrm{~d}=26.63 \AA)$ which allowed for viewing of wavelengths between 6.2 and 11.0 A, wide enough to cover most of K-shell Al and Mg spectra. Bio-max MS Kodak $\mathrm{X}$-ray film was implemented in this work. The spacing between gates was $5 \mathrm{~ns}$, while the microchannel plate was gated to last for $3 \mathrm{~ns}$. The XRD and PCD signals look very similar to that of $\mathrm{Mo} / \mathrm{Mo} / \mathrm{Mo}$, with an implosion time of $95 \mathrm{~ns}$ and many burst formations after the main implosion. Looking to the TGSP, spectra were collected starting at $79 \mathrm{~ns}$ after current rise, or $16 \mathrm{~ns}$ before the main implosion. Frame 2 in Figure 2(b) indicates the presence of background, perhaps bremsstrahlung radiation or recombination, however very weak K-shell Al radiation, and corresponds to a spike in PCD signal. This may indicate that L-shell Mo, from the outer planes, is radiating just slightly before K-shell Al, from the inner plane, however without time resolution of L-shell Mo itself this is purely speculation and points to the importance of understanding time evolution of L-shell Mo. Frame 3, which corresponds to a "valley" in the PCD signal and little background, shows definitive appearance of $\mathrm{K}$-shell Al, with an estimated $T_{e}$ of $425 \mathrm{eV}$. Plasma parameters are estimated using a non-LTE kinetic model of K-shell Al and Mg (for information of the model and labeling of important diagnostic lines, see Ref. [12]). The ratio of A12

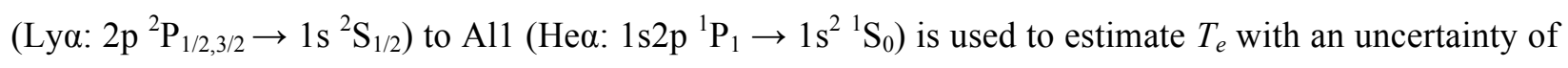
$\pm 10 \%$. Usually, the electron density is estimated by the ratio of Al1 and inter-combination line Al1' (IC: $1 \mathrm{~s} 2 \mathrm{p}^{3} \mathrm{P}_{1}-1 \mathrm{~s}^{2} \mathrm{~S}_{0}$ ) with an estimated uncertainty within an order of magnitude, however here the intensity of the Al1' is too low and the electron density $10^{20} \mathrm{~cm}^{-3}$ is used (electron densities on the order of $10^{19}-$ 
$10^{20} \mathrm{~cm}^{-3}$ are common for university scale generators, see for example Ref $\left.[13,14]\right)$. Frame 4, $5 \mathrm{~ns}$ later, which corresponds just before the main implosion and re-emergence of background, shows a K-shell Al $T_{e}$ of $430 \mathrm{eV}$. Frame 5, which corresponds to just after the main implosion, shows very intense background and a maximum of K-shell $\mathrm{Al} T_{e}$ of $450 \mathrm{eV}$. The final frame 6 corresponds to another "valley" in the PCD signal and significant reduction of background, along with reduction of K-shell Al $T_{e}$ with an estimation of $350 \mathrm{eV}$. It's important to note in this analysis that $\mathrm{K}$-shell $\mathrm{Al}$ radiated relatively optically thin, with no presence of $\mathrm{K}$-shell $\mathrm{Mg}$. This is likely do to the Al wires being in the inner plane and also starting out with a low percentage of initial mass (35\%).

Figure 3 shows the results of the $\mathrm{K}$-shell $\mathrm{Al}$ and $\mathrm{Mg}$ spectra along with signals from the $\mathrm{Al} / \mathrm{Mo} / \mathrm{Al}$ configuration. Again the XRD and PCD signals show a sharp rise followed by random bursts. The TGPH images at $>1.0 \mathrm{keV}$ in frames "c" "d" and "e", which correspond to the middle of the main implosion, are very bright and likely saturated the film, which is a contrast to the TGPH images for the $\mathrm{Mo} / \mathrm{Mo} / \mathrm{Mo}$ array, which didn't produce an oversaturation of the film. This is likely due to K-shell Al radiation from the outer planes, as will be shown in the next section when the time-integrated spatially resolved spectra are analyzed. Looking to the TGSP, similar to the previous Mo/Al/Mo case, the first frame 1 is taken before any PCD signal and no K-shell Al or Mg was measured. By frame 2, however, $\mathrm{K}$ shell Al is measured, with very weak evidence of $\mathrm{K}$-shell $\mathrm{Mg}$ and very little background on the film. The corresponding lineout indicates that K-shell Al lines All and Al2 are optically thick. Frame 3 corresponds to a rapid rise in PCD signal, strong background on the film, and strong $\mathrm{K}$-shell $\mathrm{Al}$ and $\mathrm{Mg}$ lines. Due to the high opacity of K-shell Al, K-shell $\mathrm{Mg}$ was modeled and had an estimated $T_{e}$ of $300 \mathrm{eV}$. Frames 4-6 proceed to follow the PCD signal to just before to just after the main implosion, characterized by strong $\mathrm{K}$-shell $\mathrm{Al}$ and $\mathrm{Mg}$ emissions, strong background, and modeled $\mathrm{K}$-shell $\mathrm{Mg} T_{e}$ of 325, 350, and $310 \mathrm{eV}$, respectively. As previously for Mo/Al/Mo, the modeled $T_{e}$ follows the PCD signal well.

\section{Radiative Properties of Mo and Combined with Al TPWAs}

This section will focus on the radiative properties of the TPWAs of Mo and combined with Al with a $1.5 \mathrm{~mm}$ inter-planar gap, in particular the time-integrated spatially resolved radiation, which will help in understanding axial gradients along the pinch for both K-shell Al and L-shell Mo plasmas, which is important in helping to understand plasma from different materials. Figure 4 shows the results of time-

integrated spatially resolved pinhole and spectra from $\mathrm{Mo} / \mathrm{Mo} / \mathrm{Mo}, \mathrm{Mo} / \mathrm{Al} / \mathrm{Mo}$, and $\mathrm{Al} / \mathrm{Mo} / \mathrm{Al}$. The spectra were taken with a KAP convex crystal spectrometer while the pinhole images were filtered to study L-shell Mo emissions $>3.0 \mathrm{keV}$ and $\mathrm{K}$-shell $\mathrm{Al}$ and $\mathrm{Mg}$ emission (along with L-shell Mo) > 1.0 $\mathrm{keV}$. Both diagnostics are axially resolved to study variations along the length of the pinch from anode 
"a" to cathode "c". Diagnostically important L-shell Mo lines (for more information on L-shell Mo and labeling of important diagnostic lines, along with the non-LTE kinetic model used to diagnose plasma parameters, see Ref. [15]), along with $\mathrm{K}$-shell $\mathrm{Al}$ and $\mathrm{K}$-shell $\mathrm{Mg}$ lines are indicated. The pure $\mathrm{Mo} / \mathrm{Mo} / \mathrm{Mo}$ load shows increased hard x-ray formation and less bright spot formation than the mixed loads. The mixed Mo/Al/Mo load produces optically thin $\mathrm{K}$-shell $\mathrm{Al}$ radiation with no presence of $\mathrm{K}$ shell $\mathrm{Mg}$ lines and radiates not in a column-like manner, but follows L-shell Mo radiation, which correlate with "bright" spot formations. The $\mathrm{Al} / \mathrm{Mo} / \mathrm{Al}$ load produces optically thick K-shell $\mathrm{Al}$ and has a strong presence of $\mathrm{K}$-shell $\mathrm{Mg}$ and radiates more in a column-like manner than for the $\mathrm{Mo} / \mathrm{Al} / \mathrm{Mo}$ load, though there still appears to be an influence in $\mathrm{K}$-shell $\mathrm{Al}$ and $\mathrm{Mg}$ electron temperatures wherever L-shell Mo along with "bright" spots in the film appear. For all cases, L-shell Mo radiates from many bright spots (approximately seven to ten). There are a few possible explanations to the clear change in opacity in Kshell Al lines, in particular Al1, when moving from the $\mathrm{Mo} / \mathrm{Al} / \mathrm{Mo}$ to the $\mathrm{Al} / \mathrm{Mo} / \mathrm{Al}$ configuration. The first explanation, as is explained in Ref. [5] for TPWAs of $\mathrm{Cu}$ and $\mathrm{Al}$, is that there is a current distribution between array planes, which is defined by the mutual inductance of these planes, which is affected by the load geometry rather than the specific properties of the wire materials. In short, the outer planes pick up more current than the inner planes. To illustrate this current distribution the Wire Ablation Dynamics Model (WADM [16]) was applied for configurations $\mathrm{Mo} / \mathrm{Al} / \mathrm{Mo}$ and $\mathrm{Al} / \mathrm{Mo} / \mathrm{Al}$, and results are shown in Figure 5. At $45 \mathrm{~ns}$, WADM clearly indicates most of the current is taken by the outer planes, with the inner wires of the middle plane showing little to no current. Earlier results of mixed $\mathrm{Al}$ and $\mathrm{W}$ cylindrical arrays were studied in [17] where it was shown that the Al wires imploded first, followed by the W wires, indicating an uneven current distribution due to a likely combination of inductance and resistance differences between the $\mathrm{W}$ and $\mathrm{Al}$ wires. So the current distribution is likely affected not only by placement, but also material. For more complicated loads, such as the recent star wire array configurations studied in [18], calculating and understanding current distribution is important in understanding implosion dynamics. Concerning the change in opacity in mixed arrays, similar results have been shown for $\mathrm{Al}$ and brass $(30 \% \mathrm{Zn}, 70 \% \mathrm{Cu})$ nested cylindrical wire arrays (Ref. [19]), where one cylindrical wire array was embedded in another cylindrical array. In this study, it was shown that switching the $\mathrm{Al}$ and brass wires from the inner array to the outer array dramatically affected the intensity and opacity of the $\mathrm{K}$-shell $\mathrm{Al}$ and $\mathrm{L}$-shell $\mathrm{Zn}$ and $\mathrm{Cu}$ radiation, despite having identical initial masses. The second explanation for the change in opacity could simply be due to smaller concentration of Al mass in the $\mathrm{Mo} / \mathrm{Al} / \mathrm{Mo}$ configuration compared to the $\mathrm{Al} / \mathrm{Mo} / \mathrm{Al}$ configuration.

Figure 6 shows the example of experimental lineouts (taken near the cathode in all cases) of the time-integrated spectra, as well as theoretical modeling of L-shell Mo and $\mathrm{K}$-shell $\mathrm{Al} / \mathrm{Mg}$. This is to very clearly show how different mass concentrations and wire placement can effect radiation output and that 
despite the fact that the mass of $\mathrm{Al}$ increased by twice and of Mo decreased by twice for $\mathrm{Al} / \mathrm{Mo} / \mathrm{Al}$, the ratio of $\mathrm{K}$-shell $\mathrm{Al} / \mathrm{L}$-shell Mo radiated energy went from 1 to 12 , indicating that the outer planes are likely contributing more to the total radiation then the inner plane. Non-LTE kinetic modeling was successfully applied and despite substantially different mass and plane position of Mo for each shot, the electron temperatures and densities did not significantly change for L-shell Mo: electron temperatures were estimated to be between 950 and $1200 \mathrm{eV}$ while electron densities were estimated to be between $10^{19}$ and $10^{20} \mathrm{~cm}^{-3}$. It's important to note that L-shell Mo radiated from many bright spots along the pinch, which itself indicates non-uniform plasma for L-shell Mo. The conclusion is that the bright spot formations and plasma parameters of L-shell Mo going from the $\mathrm{Mo} / \mathrm{Mo} / \mathrm{Mo}$ to $\mathrm{Mo} / \mathrm{Al} / \mathrm{Mo}$ to the $\mathrm{Al} / \mathrm{Mo} / \mathrm{Al}$ configurations do not substantially change. As for K-shell Al plasmas: electron temperatures were estimated to be between 370 and $500 \mathrm{eV}$ while electron densities were estimated to be between $6 \times$ $10^{19}$ and $5 \times 10^{20} \mathrm{~cm}^{-3}$ for $\mathrm{Mo} / \mathrm{Al} / \mathrm{Mo}$, indicating a very non-uniform implosion. For $\mathrm{Al} / \mathrm{Mo} / \mathrm{Al}, \mathrm{Mg}$ plasma temperatures were estimated to be between 300 and $360 \mathrm{eV}$ while electron densities were estimated to be between $9 \times 10^{19}$ and $2 \times 10^{20} \mathrm{~cm}^{-3}$, indicating a much more uniform implosion, yet cooler, than for $\mathrm{Mo} / \mathrm{Al} / \mathrm{Mo}$. Results of electron temperature as a function of distance from anode from these experiments are shown in Figure 7 and show the significant difference between L-shell Mo and Kshell $\mathrm{Al} / \mathrm{Mg}$ in $T_{e}$.

\section{Conclusions}

Experiments and analysis of data for triple planar wire arrays of Mo and combined with Al at 3.0 $\mathrm{mm}$ inter-planar gap and with reduced $1.5 \mathrm{~mm}$ inter-planar gap was accomplished for the first time. The reduced gap size of TPWA's ( $3 \mathrm{~mm}$ to $1.5 \mathrm{~mm}$ ) produced higher energy output (from $\sim 16 \mathrm{~kJ}$ to $\sim 25 \mathrm{~kJ}$ for pure Mo) for all configurations. Time-gated spectra modeling of K-shell Al and Mg followed PCD and XRD signals well for the $\mathrm{Mo} / \mathrm{Al} / \mathrm{Mo}$ and $\mathrm{Al} / \mathrm{Mo} / \mathrm{Al}$ configurations. The radiative properties of $\mathrm{L}-$ shell Mo and K-shell Al agree well with previous results from [3] with the investigation of mixed Mo and Al single planar wire arrays where L-shell Mo radiated from randomly generated bright spot formations between the anode and cathode. In Ref [5], $\mathrm{Cu}$ and $\mathrm{Al}$ TPWAs were tested and provide useful comparisons for the TPWAs discussed in this paper, specifically for opacity effects of mixed material plasmas. For the Mo/Al/Mo configuration, K-shell Al lines were optically thin and followed Mo L-shell radiation from bright spot pattern. This is likely due to initial placement on the inside of the array, which has less current distribution, and smaller initial Al concentration ( 35\% total mass), which is a similar observation as in [5] when an $\mathrm{Al}$ plane is put in the middle of two $\mathrm{Cu}$ planes. For the $\mathrm{Al} / \mathrm{Mo} / \mathrm{Al}$ configuration, K-shell Al lines were optically thick and followed a column-like structure. This is likely 
due to initial placement on the outside of the array, which has more current distribution, and larger initial $\mathrm{Al}$ concentration ( $\sim 70 \%$ total mass). This is interesting to compare to the result from [5] when one $\mathrm{Al}$ plane was placed on the outside of a TPWA with two $\mathrm{Cu}$ planes, where K-shell Al radiated mostly optically thin, which may confirm that initial mass concentration of a material plays a role in the overall opacity features of the plasma. In addition to the much higher electron temperature of L-shell Mo, the size of the sources is much smaller while the sizes of the sources for $\mathrm{K}$-shell $\mathrm{Al}$ and $\mathrm{L}$-shell $\mathrm{Cu}$ were comparable.

This paper focused on reporting enhanced total radiated energy as a change in geometry type and exploring the details of the results. Future experiments should investigate more of the plasma physics, such as the dynamics of the implosion and instabilities reported, to understand more of this increased radiated energy. Furthermore, while experiments from this paper and Ref [5] were accomplished with comparable currents (1.0 MA), future experiments will also explore combined material TPWAs at higher currents, to understand how these results scale with current, utilizing the Load Current Multiplier [20] (1.7 MA) on the Zebra generator. Additionally, future experiments will focus on understanding the timeevolution of L-shell Mo by utilizing a new TGSP capable of attaining spectra in the proper x-ray wavelength range $(4.0-5.5 \AA)$.

\section{Acknowledgements}

This work was supported by NNSA under DOE Cooperative Agreements DE-NA0001984 and in part by DE-FC52-06NA27616.

We would like to thank all Nevada Terawatt Facility staff that helped in attaining experimental data. We would also like to thank former UNR team members Dr. Andrey Esaulov, Dr. Kenneth Williamson, and Dr. Glenn Osborn for their contributions.

\section{References}

[1]. V.L. Kantsyrev, et al., IEEE Transactions on Plasma Science 34 (2006) 2295.

[2]. V.L. Kantsyrev, et al., Physics of Plasmas 15 (2008) 030704.

[3]. A.S. Safronova, et al., High Energy Density Physics 3 (2007) 237.

[4]. M.F. Yilmaz, et al., Journal of Quantitative Spectroscopy and Radiative Transfer 109 (2008) 2877.

[5]. A.S. Safronova, et al., Physics of Plasmas 21 (2014) 031205.

[6]. J.P. Apruzese, et al., Physics of Plasmas 8 (2001) 3799. 
[7]. D.B. Sinars, et al., Physics of Plasmas 12 (2005) 056303.

[8]. G.N. Hall, et al., Physics of Plasmas 13 (2006) 082701.

[9]. S.N. Bland, et al., Physics of Plasmas 14 (2007) 056315.

[10]. V.V. Ivanov, et al., Physics of Plasmas 20 (2013) 112703.

[11]. V. Kantsyrev, et al., Review of Scientific Instruments 72 (2001) 663.

[12]. M.F. Yilmaz, et al., High Energy Density Physics 8 (2012) 30.

[13]. M.E. Weller, et al., Physics of Plasmas 21 (2014) 031206.

[14]. I.C. Blesner, et al., Review of Scientific Instruments 80 (2009) 123505.

[15]. A.S. Safronova, et al., Physics of Plasmas 15 (2008) 033302.

[16]. A.A. Esaulov, et al., High Energy Density Physics 5 (2009) 166.

[17]. S.N. Bland, et al., AIP Conference Proceedings CP651 (2002) 83.

[18]. V.V. Ivanov, et al., Physics of Plasmas 17 (2010) 102702.

[19]. M.E. Weller, et al., High Energy Density Physics 8 (2012) 184.

[20]. A.S. Chuvatin, et al., Physical Review Special Topics - Accelerators and Beams 13 (2010) 010401.

\begin{tabular}{|c|c|c|c|c|c|c|c|}
\hline Shot \# & Material & Diameter $(\mu \mathrm{m})$ & Linear Mass $(\mu \mathrm{g} / \mathrm{cm})$ & Al (\%) & $\begin{array}{c}\text { Total Radiated } \\
\text { Energy }(\mathrm{kJ})\end{array}$ & $\begin{array}{c}\text { PCD } \\
\text { Energy }(\mathrm{J})\end{array}$ & Implosion Time (ns) \\
\hline
\end{tabular}




\begin{tabular}{|c|c|c|c|c|c|c|c|}
\hline 1261 & $\mathrm{Mo} / \mathrm{Mo} / \mathrm{Mo}$ & $7.9 / 7.9 / 7.9$ & 90 & - & 16.2 & 102 & 91 \\
\hline 1262 & $\mathrm{Mo} / \mathrm{Al} / \mathrm{Mo}$ & $7.9 / 15.0 / 7.9$ & 89 & 32 & 16.9 & 125 & 93 \\
\hline 1263 & $\mathrm{Al} / \mathrm{Mo} / \mathrm{Al}$ & $15.0 / 7.9 / 15.0$ & 87 & 65 & 13.1 & 179 & 97 \\
\hline 1969 & $\mathrm{Mo} / \mathrm{Mo} / \mathrm{Mo}$ & $8.9 / 8.9 / 8.9$ & 114 & - & 25.5 & 157 & 94 \\
\hline 1932 & $\mathrm{Mo} / \mathrm{Mo} / \mathrm{Mo}$ & $8.9 / 8.9 / 8.9$ & 114 & - & 24.5 & 158 & 99 \\
\hline 1968 & $\mathrm{Mo} / \mathrm{Al} / \mathrm{Mo}$ & $8.9 / 17.8 / 8.9$ & 116 & 35 & 22.2 & 220 & 95 \\
\hline 1933 & $\mathrm{Mo} / \mathrm{Al} / \mathrm{Mo}$ & $8.9 / 17.8 / 8.9$ & 116 & 35 & 22.5 & 274 & 97 \\
\hline 1953 & $\mathrm{Al} / \mathrm{Mo} / \mathrm{Al}$ & $17.8 / 8.9 / 17.8$ & 119 & 68 & 23.1 & 349 & 95 \\
\hline 2166 & $\mathrm{Al} / \mathrm{Mo} / \mathrm{Al}$ & $17.8 / 8.9 / 17.8$ & 119 & 68 & 24.0 & 301 & 97 \\
\hline
\end{tabular}

TABLE 1. List of considered shots and parameters for Mo and combined Mo and Al TPWAs, with load characteristics, diameter of the wires, inter-planar gap, linear mass, percentage of Al in the load, PCD energy, and total radiated energy. The first three listed are loads with inter-planar gap of $3.0 \mathrm{~mm}$. The last six are loads with inter-planar gap of $1.5 \mathrm{~mm}$.
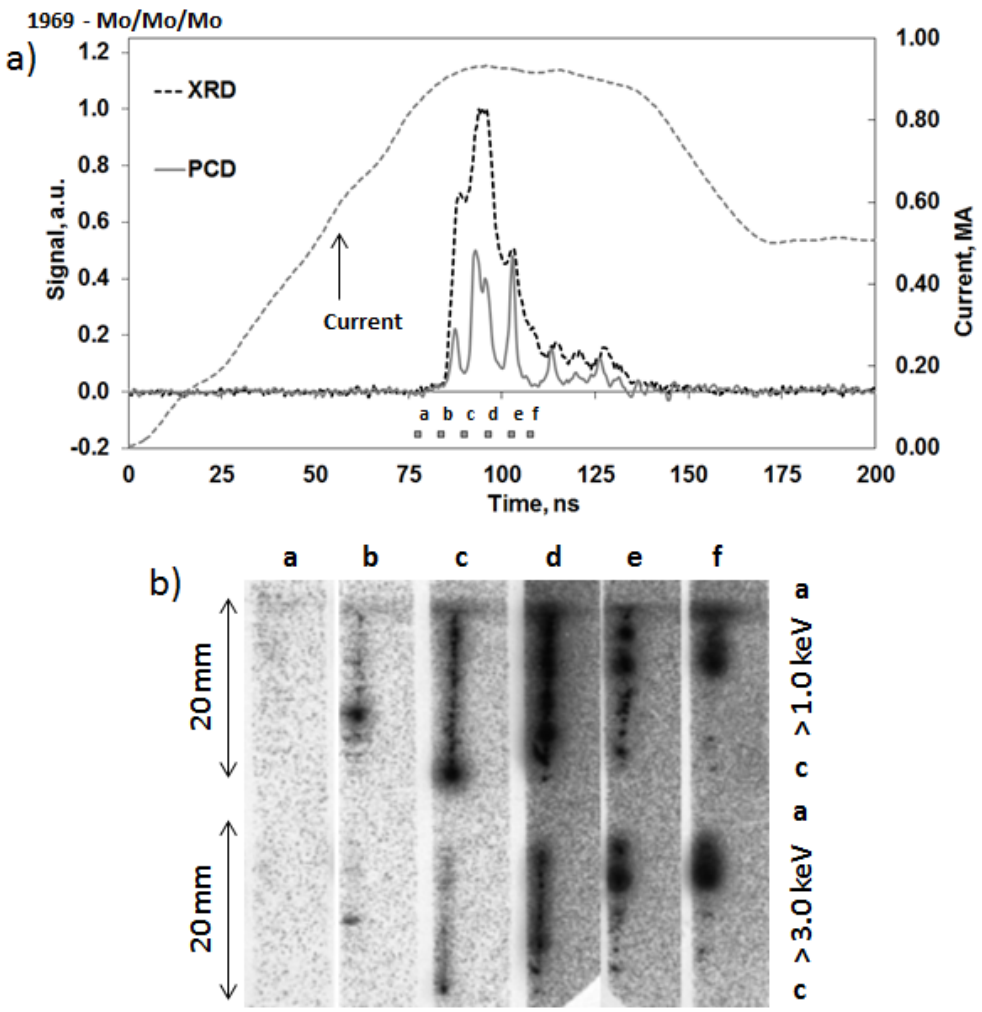

FIGURE 1. (a) PCD and XRD signals along with current (b) and time-gated pinhole images for $\mathrm{Mo} / \mathrm{Mo} / \mathrm{Mo}$ (1969). The pinhole timings are shown with gray boxes and are listed "a-f" and are filtered at $>1.0 \mathrm{keV}$ and $>3.0 \mathrm{keV}$. 


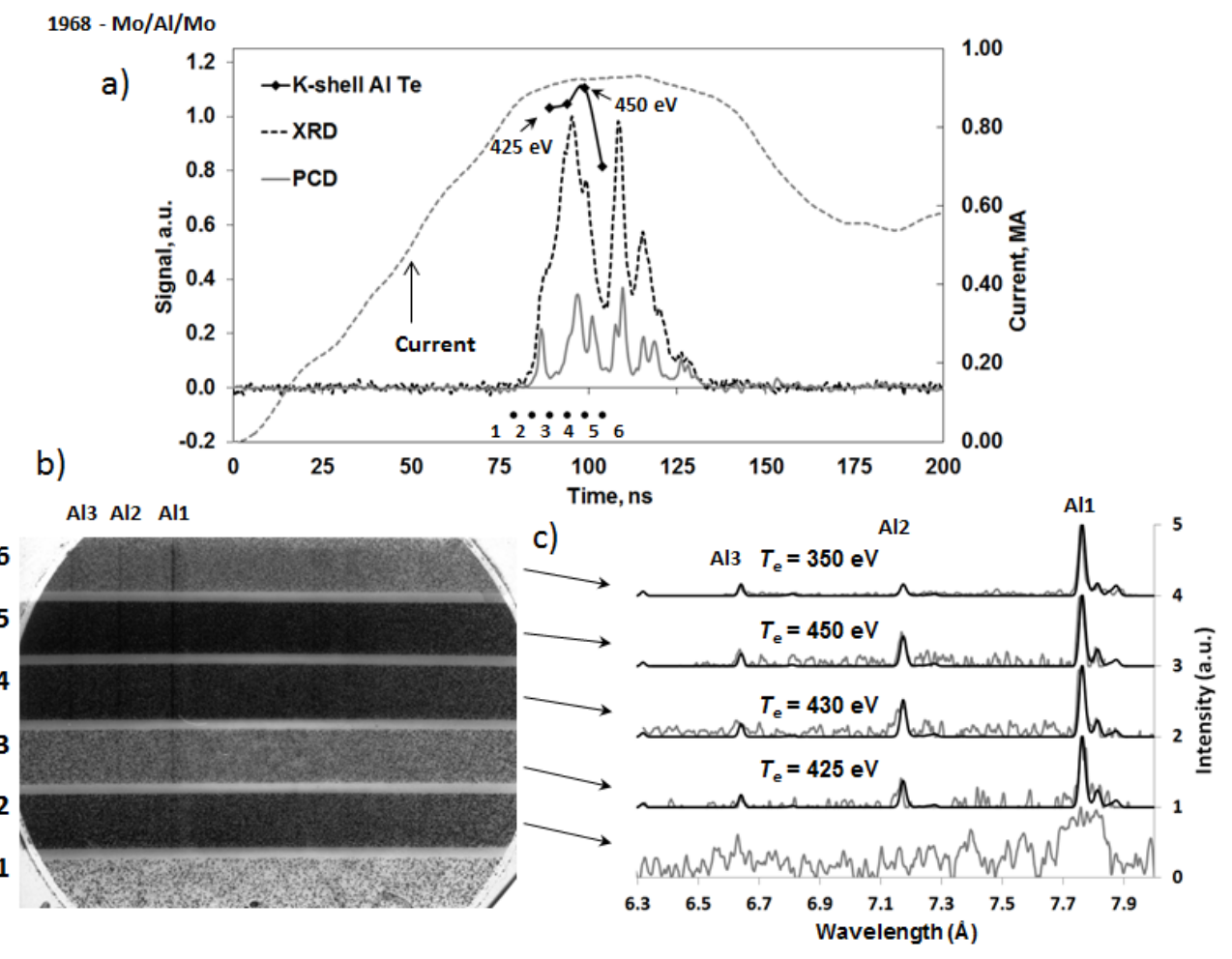

FIGURE 2. (a) PCD and XRD signals from Mo/Al/Mo along with current, timings from the time-gated spectrometer (labeled "1 - 6"), and K-shell Al electron temperatures. (b) Spectra from time-gated spectrometer and (c) lineouts taken from spectra in (b) along with synthetic spectra. 

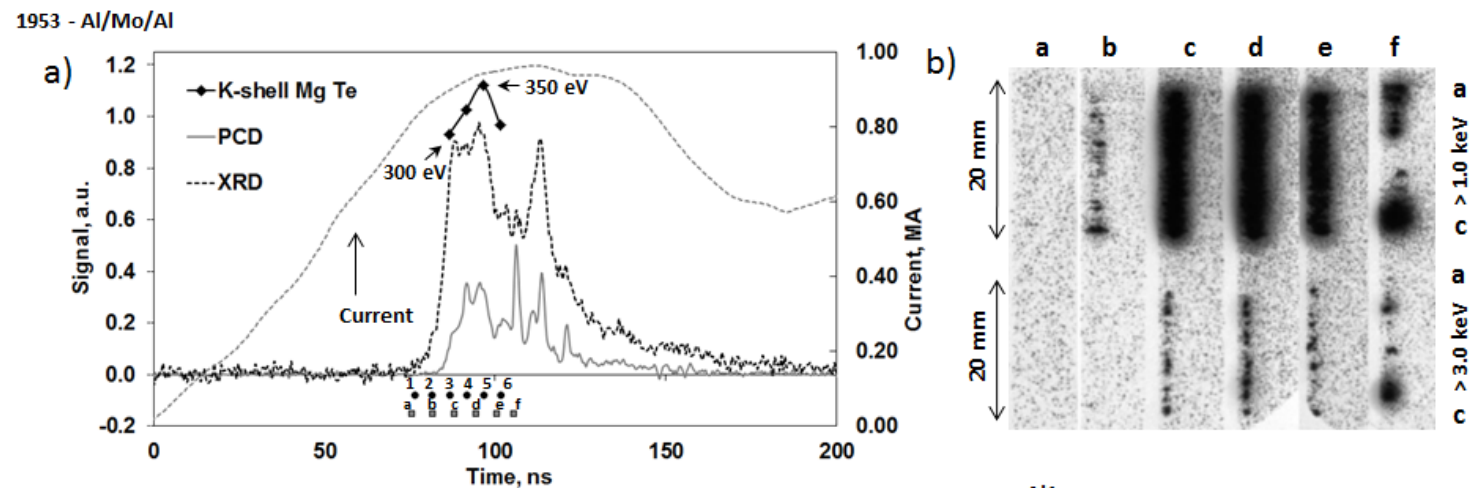

c)
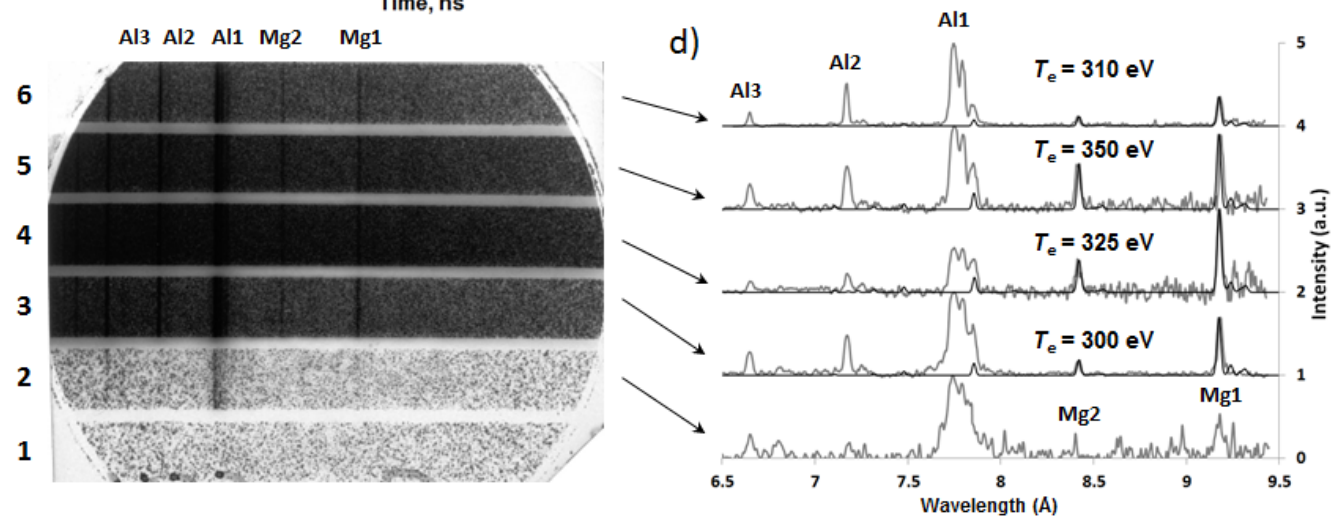

FIGURE 3. (a) $\mathrm{PCD}$ and $\mathrm{XRD}$ signals from $\mathrm{Al} / \mathrm{Mo} / \mathrm{Al}$ along with current, timings from the time-gated spectrometer (labeled " $1-6$ "), and K-shell Al electron temperatures. (b) Time-gated pinhole images. The pinhole timings are shown with gray boxes and are listed "a-f" and are filtered at $>1.0 \mathrm{keV}$ (top) and > $3.0 \mathrm{keV}$ (bottom). (c) Spectra from time-gated spectrometer and (d) lineouts taken from spectra in (c) along with synthetic spectra. 


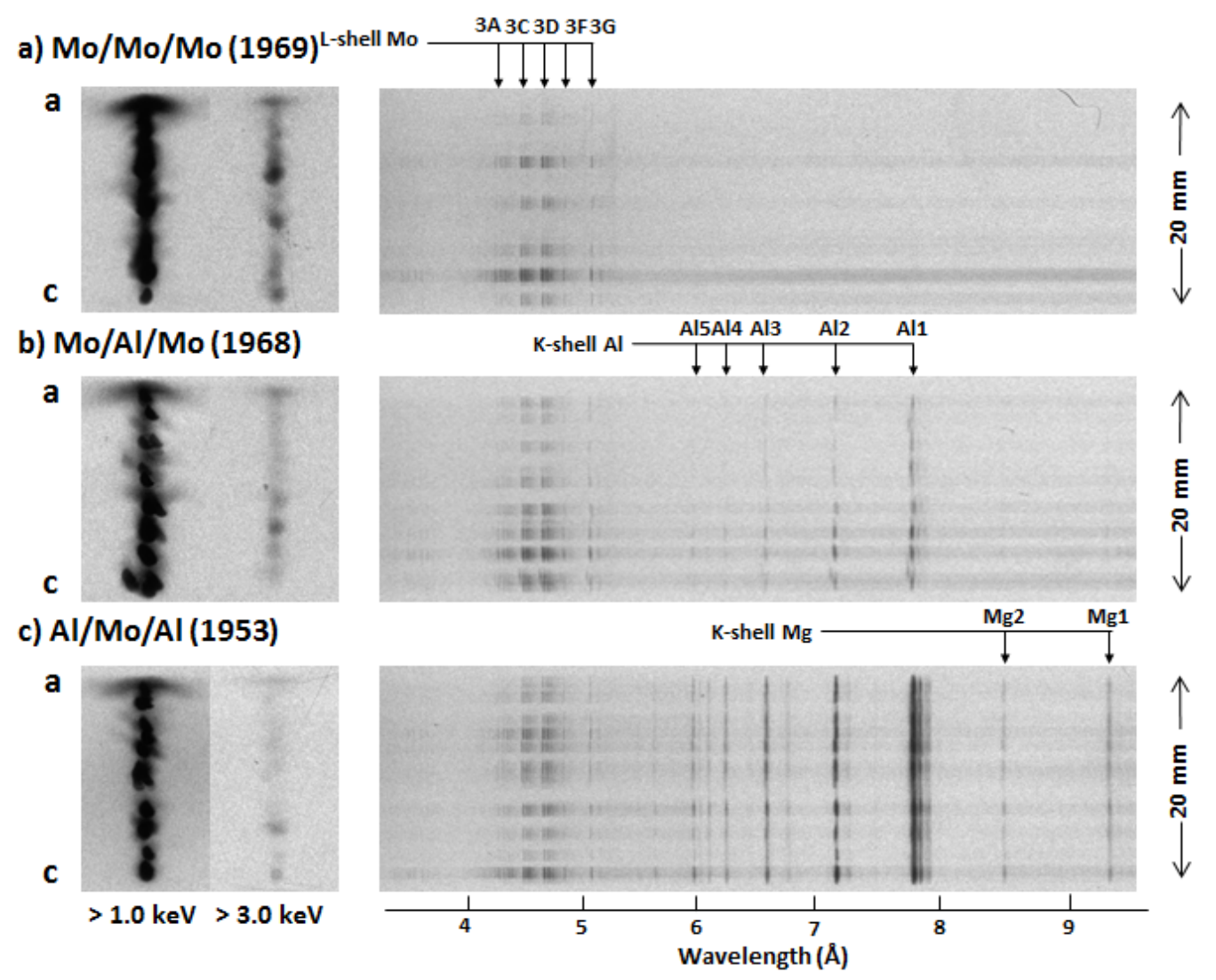

FIGURE 4. (a) Time-integrated spatially resolved pinholes (left) and spectra (right) of $\mathrm{Mo} / \mathrm{Mo} / \mathrm{Mo}$. (b) Time-integrated spatially resolved pinholes (left) and spectra (right) of $\mathrm{Mo} / \mathrm{Al} / \mathrm{Mo}$. (c) Time-integrated spatially resolved pinholes (left) and spectra (right) of $\mathrm{Al} / \mathrm{Mo} / \mathrm{Al}$. 

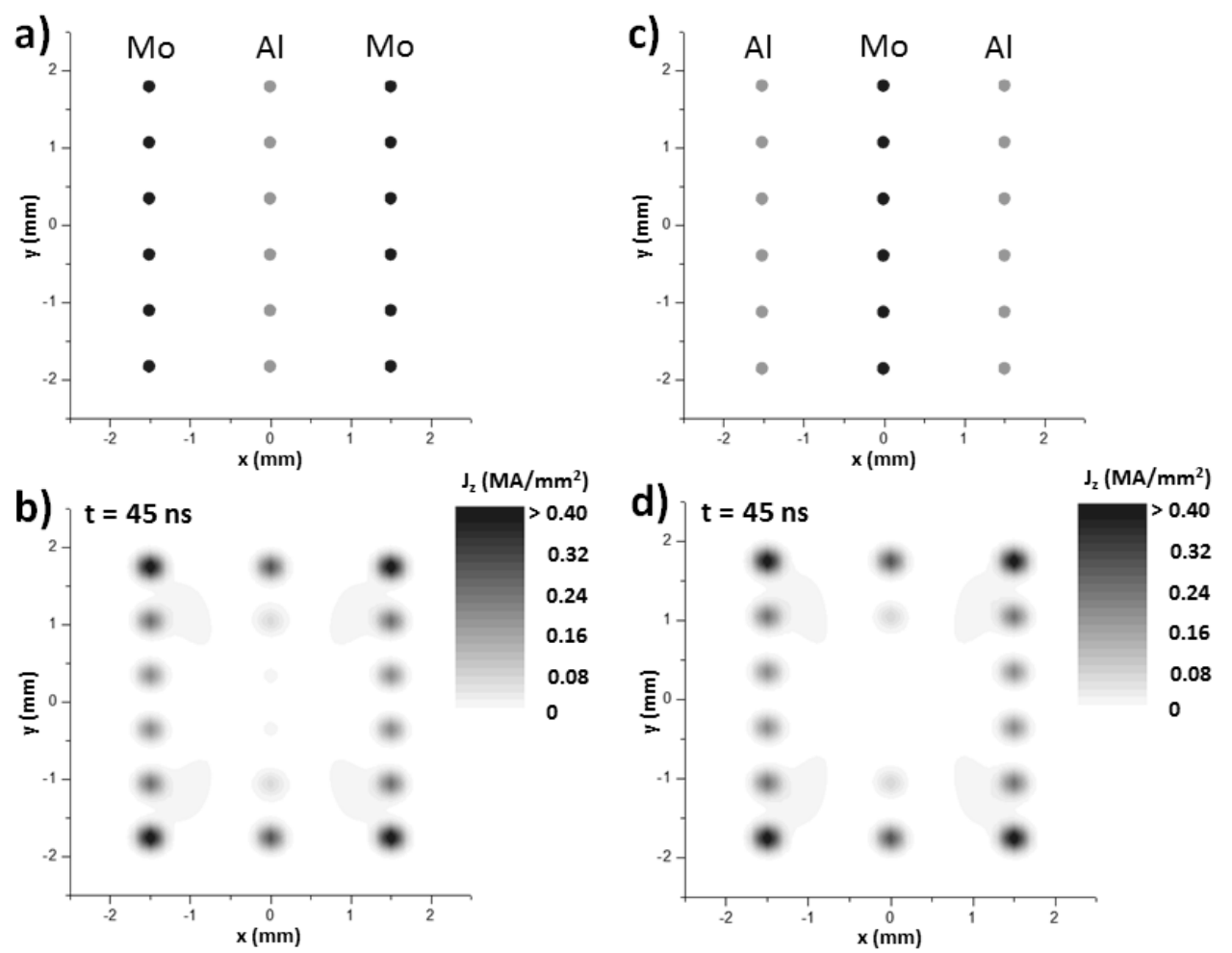

FIGURE 5. Wire Ablation Dynamics Modeling for configurations $\mathrm{Mo} / \mathrm{Al} / \mathrm{Mo}$ and $\mathrm{Al} / \mathrm{Mo} / \mathrm{Al}$. (a) The initial wire position for $\mathrm{Mo} / \mathrm{Al} / \mathrm{Mo}$ and (b) the current distribution at $\mathrm{t}=45 \mathrm{~ns}$. (c) The initial wire position for $\mathrm{Al} / \mathrm{Mo} / \mathrm{Al}$ and (d) the current distribution at $\mathrm{t}=45 \mathrm{~ns}$.

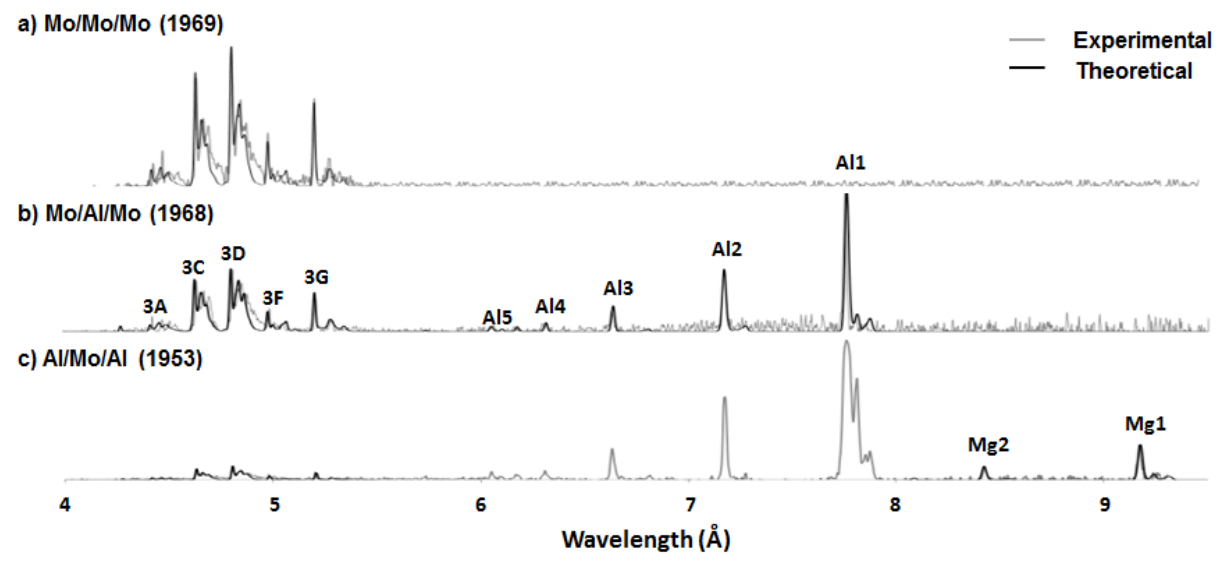

FIGURE 6. Lineouts in gray taken near the cathode from time-integrated spatially resolved spectra from Figure 4 of (a) Mo/Mo/Mo, (b) Mo/Al/Mo, and (c) Al/Mo/Al. Theoretical modeling is shown in black. 


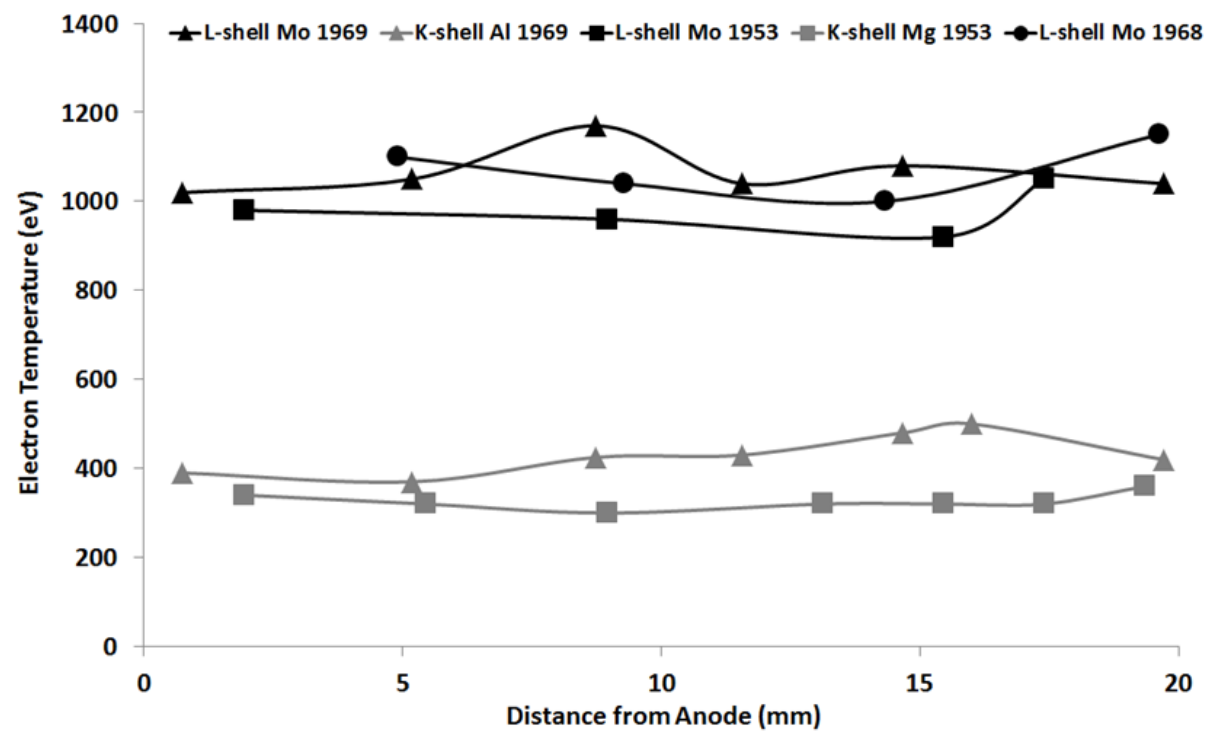

FIGURE 7. Modeled electron temperature as a function of distance from anode of L-shell Mo and Kshell Al/Mg from Zebra shots 1968 (Mo/Mo/Mo), 1969 (Mo/Al/Mo), and 1953 (Al/Mo/Al). 